\title{
LA PUISSANCE FRANÇAISE SOUS LE FRONT POPULAIRE : LE REGARD DES BELGES FRANCOPHONES
}

\section{Catherine Lanneau}

\section{Presses Universitaires de France | « Guerres mondiales et conflits contemporains »}

$2004 / 3 n^{\circ} 215$ | pages 75 à 89

ISSN 0984-2292

ISBN 2130547214

\section{Article disponible en ligne à l'adresse :}

http://www.cairn.info/revue-guerres-mondiales-et-conflits-contemporains-2004-3-page-75.htm

\section{!Pour citer cet article :}

Catherine Lanneau, « La puissance française sous le Front populaire : le regard des Belges francophones », Guerres mondiales et conflits contemporains 2004/3 (n²15), p. 75-89. DOI 10.3917/gmcc.215.0075

Distribution électronique Cairn.info pour Presses Universitaires de France.

(C) Presses Universitaires de France. Tous droits réservés pour tous pays.

La reproduction ou représentation de cet article, notamment par photocopie, n'est autorisée que dans les limites des conditions générales d'utilisation du site ou, le cas échéant, des conditions générales de la licence souscrite par votre établissement. Toute autre reproduction ou représentation, en tout ou partie, sous quelque forme et de quelque manière que ce soit, est interdite sauf accord préalable et écrit de l'éditeur, en dehors des cas prévus par la législation en vigueur en France. Il est précisé que son stockage dans une base de données est également interdit. 


\section{LA PUISSANCE FRANÇAISE SOUS LE FRONT POPULAIRE : LE REGARD DES BELGES FRANCOPHONES}

Ce n'est ni faire injure à la France ni exprimer une opinion révolutionnaire que d'associer l'idée de déclin, d'affaiblissement, à sa politique extérieure des années 1930. Le maître de l'histoire diplomatique, Jean-Baptiste Duroselle, ne parle-t-il pas lui-même de "décadence " ${ }^{1}$ ? Les Belges francophones, immergés dans l'atmosphère française, ressentent douloureusement cette perte de prestige et voient avec angoisse l'Allemagne hitlérienne en profiter tandis que leur pays parachève, par le discours royal du 14 octobre 1936, son inexorable évolution vers une politique d'indépendance ${ }^{2}$. Au cœur de cette période charnière, la France se dote d'un gouvernement de Front populaire ${ }^{3}$ dont les actes vont être analysés avec plus de passion que d'objectivité. Le jugement de l'opinion belge francophone sur la politique intérieure répond, on s'en doute, au clivage gauche-droite. Mais, la politique étrangère, traditionnellement moins fluctuante, plus consensuelle, entraîne-t-elle le même raidissement idéologique? Quel rôle attribue-t-on au Front populaire dans l'érosion de la puissance française ${ }^{4}$ ?

\section{LA DROITE ET LE CENTRE ENTRE A PRIORI ET NUANCES}

Les premières semaines qui suivent la victoire de la gauche voient la presse conservatrice tonner contre un effondrement subit du prestige fran-

1. J.-B. Duroselle, Politique étrangère de la France. La décadence 1932-1939, Paris, 1979. Dans l'historiographie anglo-saxonne, voir surtout R. W. D. Boyce, French Foreign and Defence Policy 1918-1940 : The Decline and Fall of a Great Power, Londres-New York, 1998.

2. Voir notamment C. Koninckx, Léopold III roi et diplomate. La politique extérieure belge et les initiatives de paix pendant l'entre-deux-guerres (1934-1940), Anvers, 1997.

3. Nous entendrons par Front populaire la période allant de mai 1936 à la formation, en avril 1938, d'un cabinet Daladier qui enterre le front des gauches.

4. Sur la puissance française à la fin des années 1930, voir R. Girault et R. Frank (dir.), La puissance en Europe 1938-1940, Paris, 1984, et le numéro spécial de Relations internationales : "Images de la France en 1938-1939", n 33, printemps 1983. 
çais, attribué aux grèves et à l'agitation révolutionnaire. La Gazette, libérale de droite et volontiers antiparlementariste, signale que, durant tout ce mois de mai 1936 où la France " en relâche $"^{5}$ a attendu un nouveau gouvernement, les soldats allemands n'ont pas ralenti leur progression en Rhénanie réoccupée et qu'il sera bien difficile de les en déloger. Des libéraux moins conservateurs dénoncent également les conséquences néfastes du Front populaire. Pour la revue Le Flambeau, proche de l'Université Libre de Bruxelles, Paris entre dans "l'ère de la parfaite inconscience $"^{6}$. De l'autre côté du Rhin, l'Allemagne "observe attentivement l'état intérieur de la France et suppute la diminution d'activité, de prestige et d'influence qui en serait la conséquence $»^{7}$. Dans toutes les capitales plus ou moins alliées, dont Bruxelles, on se détournera de Paris, au moins provisoirement. Le Flambeau lance dès lors un avertissement : "Nous tenons à dire à nos amis français que la politique du pire pratiquée en ce moment $[\ldots]$ comporte des risques redoutables, non seulement pour la paix intérieure et l'économie française [...] mais aussi, et cela immédiatement, pour la situation de la France en Europe. $»^{8}$

Les conservateurs soulignent l'évolution en sens contraire des États forts et de la France. Le journal catholique namurois Vers l'Avenir estime que celle-ci est devenue une puissance de seconde zone, expression promise à un bel avenir dans la presse de droite. "Elle ne peut opposer à la forte unité de ses rivales que les discordes civiles et la désagrégation sociale. Voilà les lauriers de M. Blum ! Voilà l'œuvre du Front populaire en France. " Insistant davantage sur l'idée d'une dégradation entamée depuis la guerre mais soudain patente, le quotidien de Léon Degrelle, le Pays Réel relève avec complaisance les titres des presses fasciste et hitlérienne: "La France se désagrège, la France se décompose, c'est une nation désormais de deuxième ordre. $»^{10}$

Durant tout le moins de juin 1936, La Nation Belge, proche des idées maurrassiennes, publie plusieurs articles, envoyés de Berlin par son correspondant, l'Alsacien Georges Blun. L'idée fondamentale de ces chroniques est de décrire une France hors jeu, rayée de la carte diplomatique. «Évidemment, on ne regarde pas encore la France par-dessus l'épaule mais on la tient déjà en moindre estime. On la considère comme la victime d'un processus de désagrégation nationale qui la rend moins apte à jouer en Europe le rôle qu'elle y a joué jusqu'ici. $»^{11}$ Les Allemands, ajoute Blun, ressentent parfois de la compassion en voyant "un si beau pays abandonné sans direction aux vagues hurlantes de la tempête rouge.

5. Gazette (ci-après Gaz), 31 mai 1936, p. 1.

6. Flambeau, juin 1936, p. 749.

7. Ibid., juillet 1936 , p. 110.

8. Ibid., p. 112.

9. Vers l'Avenir (ci-après VA), 16 juillet 1936, p. 1.

10. Pays Réel (ci-après $P R), 12$ juin 1936, p. 1.

11. La Nation Belge (ci-après NB), 9 juin 1936, p. 1 
Ils ont compris que la politique extérieure française serait plus mièvre que jamais, sans objectif précis $»^{12}$. On pourrait difficilement dresser un tableau plus sombre.

Au fil des semaines, la droite conjugue, sous toutes ses formes, le thème de l'isolement de la France : les petites nations alliées n'ont plus confiance en elle, les grandes puissances européennes la tiennent à l'écart. La Revue catholique des idées et des faits, sous la plume de l'écrivain britannique Hilaire Belloc, estime également que le facteur primordial du trouble européen se situe en France, dans la mesure où celle-ci s'affaiblit par son incapacité défensive comme par son absence de décision. Au-delà du Front populaire, c'est au régime que s'adressent ses critiques. La constitution de la France la prive des éléments d'ordre et de continuité nécessaires à une politique étrangère digne de ce nom : un centre de décision, une monarchie, une classe dirigeante. "Le pays ne peut continuer comme il va avec un vieux Juif dilettante comme chef nominal et une émulation d'intérêts particuliers prenant la place de l'Autorité $»^{13}$, écrit-il. Le même argument xénophobe est, évidemment, exploité par la presse rexiste. Pour Hubert d'Ydewalle, dans Le Pays Réel, le catalyseur qui rend soudain la décomposition de la France plus rapide est l'invasion étrangère et sémitique. "Le destin des peuples va vite. Il y a quinze ans, la France était encore considérée comme une grande puissance. Elle avait déjà en elle des germes terribles de corruption. Mais, elle était encore, comme par hasard, gouvernée par des Français. On la respectait un peu par habitude. ${ }^{14}$ Comme dans la presse française, Léon Blum et son entourage sont ici virulemment pris pour cibles ${ }^{15}$.

Cependant, à partir de l'automne 1936, certains conservateurs vont parfois délaisser leurs préventions idéologiques pour comparer la politique étrangère du Front populaire à celle des cabinets précédents. Ainsi, Vers l'Avenir publie, la même semaine, deux articles presque contradictoires. Tandis que sa rédaction namuroise fustige une "France sans prestige, désarmée ${ }^{16}$, son envoyé spécial à Paris, Jean de Moreau, fait paraître une mise au point contrastée sur la diplomatie dans "la France de M. Blum ». Il décrit le président du Conseil comme un héritier et note que, si sa présence avive les inquiétudes au sujet du Pacte franco-soviétique, son rôle réel est passif ${ }^{7}$. Le Front populaire français a bien réagi face à la guerre d'Espagne en prônant la non-intervention, il a réussi à se rapprocher de la Grande-Bretagne et il vote les crédits d'armement nécessaires. Dans l'ensemble, conclut de Moreau, la politique extérieure de Blum est modératrice: il abandonne les théories socialistes pour faire face aux

12. Ibid., 27 juin 1936, p. 1.

13. Revue catholique des idées et des faits, 9 octobre 1936, p. 18.

14. PR, 16 septembre 1936, p. 1.

15. Sur l'atmosphère française, nous renvoyons surtout à Birnbaum (P.), Un mythe politique : "La République juive », Paris, 1988.

16. $V A, 19$ février 1937, p. 1.

17. $V A, 25$ février 1937, p. 1. 
réalités ${ }^{18}$. Même si cet article ne reflète manifestement pas l'opinion de la rédaction, elle l'a publié sans commentaires.

Par ailleurs, tous les protagonistes du Front populaire ne représentent pas le même danger. Les personnalités d'Yvon Delbos, ministre des Affaires étrangères, et d'Édouard Daladier, ministre de la Défense nationale et de la Guerre, tous deux radicaux, sont épargnées par les critiques les plus virulentes. On souligne fréquemment que Daladier s'acquitte en conscience de sa mission. Il veille à ce que les idéaux bolcheviques ne pénètrent pas l'armée, comme le remarque le maurrassien français Pierre Gaxotte dans La Nation Belge ${ }^{19}$, et ne peut être accusé de tous les maux sur la question du déficit des armements. D'autre part, La Gazette absout Blum mais surtout Daladier pour "l'état défectueux » de l'armée française, arguant que «le véritable coupable, c'est le régime parce qu'il n'a su ni prévoir, ni ordonner $»^{20}$. En ce qui concerne Delbos, La Gazette estime qu'il est injuste de lui attribuer l'isolement de la France : "Ses prédécesseurs ont souvent été moins bien inspirés ${ }^{21}$, note-t-elle en rappelant les erreurs de Sarraut et Flandin lors de la remilitarisation de la Rhénanie. Et Gaxotte d'indiquer, dans La Nation Belge, que le ministre des Affaires étrangères est passé maitre dans l'art de louvoyer et d'éviter le pire ${ }^{22}$. Bref, la politique étrangère du Front populaire ne peut être rejetée en bloc.

Au centre, on la considère plutôt comme un moindre mal. Les décisions prises sont acceptables et n'engendrent pas un affaiblissement particulier de la France. Dans Le Soir, le billet politique quotidien est rédigé depuis Paris où son auteur, Roland de Marès, est rédacteur au Temps. Il a toujours reflété l'opinion du Quai d'Orsay, et l'arrivée du Front populaire ne change pas la donne. Dès les premiers jours, il insiste sur le fait qu'aucune rupture réelle ne doit être attendue car les faits dominent et s'imposent à tous. "Le gouvernement de Front populaire marque son désir d'assurer la continuité de la politique extérieure de la France telle que l'ont fixée les gouvernements républicains bourgeois qui se sont succédé au pouvoir à Paris depuis la fin de la guerre. $»^{23}$

Dans les mois qui suivent, les billets insistent sur le rôle modérateur et bénéfique des radicaux, tels $\operatorname{Delbos}^{24}$ et Daladier ${ }^{25}$, qui assurent la pérennité de la diplomatie française et de son armée, protégeant le pays de tout excès. Ils déploient également une énergie évidente à nier l'idée d'un déclin de la France : la politique du Quai d'Orsay jouit, selon eux, d'une armature solide, capable de rapprocher les diverses nations sans en exclure aucune. "Tout le reste n'est qu'illusion ou fantaisie de gens qui spéculent

18. Ibid., 26 février 1937, p. 1.

19. NB, 15 mars 1937, p. 1.

20. Gaz, 30 janvier 1937, p. 1.

21. Gaz, $1^{\mathrm{er}}$ mai 1937 , p. 1 .

22. NB, 15 mars 1937, p. 1.

23. Soir, 25 juin 1936, p. 1.

24. Ibid., 29 novembre 1936, p. 1.

25. Ibid., 24 avril 1937, p. 1. 
avec autant de témérité que de naïveté sur un isolement de la France en Europe, isolement qui n'existe pas, qui n'a jamais existé, ne saurait exister, par la nature même des intérêts permanents et vitaux des peuples du continent. ${ }^{26}$ Toutefois, les premiers mois de 1938 mettront un terme à cet optimisme.

La catholique Revue générale, très sévère sur la politique intérieure française, juge qu'en ce qui concerne la diplomatie, la panique s'est emparée à tort de l'opinion. "On craignait, et non sans apparence de raison, que ce cabinet d'extrême gauche n'entamât une croisade des démocraties contre les fascismes. Il n'en a rien été. ${ }^{27} \mathrm{La}$ revue estime que le Front populaire œuvre au réarmement de la France et il associe le président du Conseil à l'hommage qu'il rend à Édouard Daladier. "C'est l'honneur de M. Léon Blum d'avoir mis au grenier ses préjugés antimilitaristes pour apporter à l'organisation militaire de la France le même zèle qu'aurait pu y mettre n'importe quel gouvernement nationaliste. " ${ }^{28}$

Du côté libéral, l'hebdomadaire francophile Pourquoi Pas $?^{29}$ comme le quotidien radical La Dernière Heure, louent les mérites de Delbos. Pour l'avocat Henry van Leynseele, il « est, par la loyauté et le bon sens, infiniment supérieur à ses prédécesseurs immédiats et il faut remonter à Briand pour rencontrer un homme ayant, au même point, une juste vision des intérêts véritables de son pays et de l'Europe $»^{30}$. Mais, La Dernière Heure salue également l'action de Blum, tant sur l'Espagne que sur le désarmement. Cette appréciation très positive s'explique, entre autres, par l'amélioration des relations franco-britanniques. Le quotidien libéral, partisan des choix politiques anglais, s'enthousiasme pour ceux du Front populaire dans la mesure où ils s'en inspirent.

Dans la catholique Libre Belgique, très attachée à la SDN et à la sécurité collective, les premières déclarations de Léon Blum en tant que président du Conseil sont plutôt bien accueillies. Pour le Français Joseph Ageorges, proche des démocrates populaires, parler de désarmement et de paix indivisible en ces temps troublés est un signe de courage et il juge que le président du Conseil "s'est exprimé en honnête homme " ${ }^{31}$. Paul Struye, futur ministre et principal chroniqueur de politique étrangère, a d'autant moins de mal à adopter la politique suivie qu'elle est approuvée en France par une partie du centre-droit et suscite l'ire des communistes ${ }^{32}$.

Trois éléments primordiaux expliquent l'apaisement de l'automne 1936. Sur le plan social, la France traverse alors une période d'accalmie relative après l'été de tous les dangers. La vague de grèves de mai-juin est désormais résorbée et les mouvements sociaux qui subsistent

26. Ibid., $1^{\text {er }}$ septembre 1936 , p. 1.

27. $R G$, octobre 1936 , p. 400.

28. Ibid., avril 1937 , p. 501.

29. PP, 26 février 1937 , p. 658.

30. La Dernière Heure, 17 janvier 1937, p. 1.

31. Libre Belgique (ci-après LB), 4 juillet 1936, p. 1.

32. Ibid., 8 décembre 1936, p. 1. 
sont sporadiques. La droite, qui avait cru au "Grand Soir ", pousse un soupir de soulagement puisque la vague rouge n'a ni submergé la France, ni surtout franchi la frontière belge. Par une forme d'extension logique, cette détente interne semble insuffler plus de nuances dans le jugement porté sur l'action internationale du Front populaire.

D'autre part, la guerre d'Espagne a pesé de tout son poids. Si les organes les plus conservateurs, comme La Nation Belge ou Le Pays Réel, ne cesseront jamais réellement de relayer les plus polémiques de leurs confrères français - Candide ou Gringoire sont largement diffusés en Belgique en accusant Blum de soutenir en sous-main les républicains, la décision française de non-intervention sera très majoritairement saluée et donnera du cabinet de Front populaire l'image d'un gouvernement responsable, sachant faire passer l'intérêt de la France avant sa fidélité idéologique. La Libre Belgique, La Revue Générale, Vers l'Avenir, tous trois catholiques, mais également Le Flambeau ou Pourquoi Pas ?, du côté libéral, sont autant d'organes peu suspects de sympathies pour le "Frente popular " et qui louent la politique espagnole de Paris.

Enfin, le 14 octobre marque une rupture dans le regard porté sur la France par l'opinion belge. Avant que Léopold III n'affirme officiellement le désir d'indépendance de la Belgique, l'opinion, qu'elle s'en réjouisse ou qu'elle le déplore avec virulence, vivait sur la conception héritée de l'accord militaire franco-belge de 1920 qui, obsolète dès la conférence de Locarno en 1925, ne fut pourtant dénoncé formellement qu'en mars $1936^{33}$. Dans cette optique, l'acharnement mis à vilipender la voisine du sud pouvait se comprendre comme un appel poignant au redressement ou comme un argument massue en faveur du retour à la neutralité. Une fois l'enjeu dépassé par les événements, le combat a presque naturellement perdu de son intensité.

L'ambassade de France à Bruxelles a finement perçu cette évolution de l'opinion publique. Le 11 août 1936, le chargé d'affaires Armand Gazel envoie un rapport alarmant au Quai d'Orsay ${ }^{34}$. Il y détaille les sentiments et les réactions que la France suscite dans les milieux belges les plus divers. S'il signale, certes, que le déclin de l'amitié franco-belge s'est amorcé dès 1934, il n'en est pas moins sévère pour les dirigeants actuels. "L'avènement d'un gouvernement de Front populaire, à Paris, malgré les sentiments pacifiques qui l'animent, n'a fait que renforcer ici les craintes nées du pacte franco-soviétique. [...] Ces appréhensions se sont encore accrues depuis que la guerre civile espagnole a engendré des possibilités de conflit international. " $^{35}$ Et d'ajouter, désabusé : «Ce n'est plus que dans le Parti socialiste et le Parti communiste que nous pouvons présentement

33. Les relations militaires franco-belges de mars 1936 an 10 mai 1940. Travaux d'un colloque d'historiens belges et français, Paris, 1968, p. 17-42 ; G. Provoost, Vlaanderen en het militair-politiek beleid in België tussen de twee wereldoorlogen, Louvain, 1976-1977, 2 t.

34. Documents diplomatiques français (1932-1939), $2^{\text {e }}$ série (ci-après DDF), t. III, Paris, 1966, p. $182-191$

35. DDF, t. III, p. 183. 
compter sur un appui solide et discipliné. ${ }^{36}$ Cette dernière remarque est à la fois trop optimiste, comme nous le verrons, et trop pessimiste puisqu'elle occulte la francophilie toujours fidèle de certains milieux libéraux francophones et des militants du mouvement wallon. Bien que minoritaires, ces deux tendances ne peuvent être négligées.

En septembre, déjà, une amélioration est perceptible. L'ambassadeur Jules Laroche s'entretient de la politique extérieure française avec le ministre des Affaires étrangères Paul-Henri Spaak. "Comme je m’y attendais - écrit-il -, M. Spaak m'en a fait le plus vif éloge et il en a souligné la modération et le courage et m'a assuré qu'elle répond entièrement aux vues du gouvernement belge. $»^{37}$ La suite de la dépêche insiste sur la campagne anti-française qui n'en continue pas moins de se développer en Belgique, Laroche et Spaak s'accordant à dire qu'une amélioration de la situation intérieure française aurait des conséquences salutaires. L'avenir semble leur donner raison. Le 13 février 1937, Laroche écrit : «On commence à sentir, dans la presse et dans les milieux officieux, une réelle détente dans les sentiments professés à notre égard, depuis que la situation intérieure en France et la situation extérieure consécutive aux affaires d'Espagne s'avèrent favorables. " $^{38}$

Néanmoins, la charnière 1937-1938 amène un revirement certain. La droite conservatrice et le centre vont fustiger l'instabilité gouvernementale qui fragilise la France alors qu'Hitler a lancé la course à l'Anschluss. Le jour où le Reich annexe l'Autriche, Paris est sans gouvernement... La catholique Gazette de Liège s'insurge : "Les patriotes de France ont fait la constatation effarante que leur pays n'est plus à la hauteur de sa mission historique. Le Front populaire a tout désorganisé. " ${ }^{39}$ La Nation Belge et Le Pays Réel, pour leur part, inscrivent davantage le déclin français dans une continuité. "L'opposition saute aux yeux: Allemagne en train de devenir le pivot de l'Europe ; France au dos courbé, dans une solitude complète. Qui eût prévu cela le 11 novembre 1918, quand les clairons sonnaient l'Armistice? Il suffit de politiciens d'un côté et d'un chef de l'autre côté pour que l'histoire soit retournée $\star^{40}$, écrit le quotidien rexiste, de plus en plus germanophile. Dans La Nation Belge, l'écrivain Louis Dumont-Wilden se livre à une "méditation dans l'angoisse ». Un ami français pleure avec lui la France "glorieuse et prestigieuse des années 1920. Les sociétés, voyez-vous, sont comme le fromage de Camembert. Il n'est parfait, exquis qu'au moment où il va se décomposer et sentir l'ammoniaque $»^{41}$.

Plus au centre, Pourquoi Pas ? brossait déjà un bilan pessimiste en janvier 1938 : «La France a la meilleure armée du monde mais elle n’a pas de

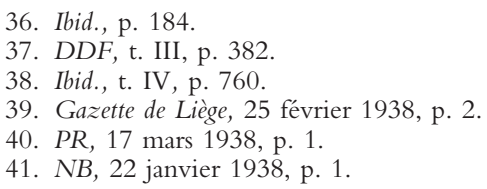


gouvernement stable et elle n'a plus guère de finances. ${ }^{42}$ Quelques jours avant l'Anschluss, La Libre Belgique relève également que "la faiblesse de la France, le déclin de son prestige dans le monde sont dus, pour une large part, à ses querelles intestines $»^{43}$.

Cependant, c'est sans conteste l'annexion de l'Autriche qui révèle au monde l'impuissance des nations démocratiques et, en particulier, de la France. Pour les États orientaux auxquels elle est liée depuis les années 1920, à savoir la Pologne, la Tchécoslovaquie, la Roumanie et la Yougoslavie, la désillusion est profonde ${ }^{44}$. Une fois passés les premiers instants de stupeur, La Dernière Heure envisage la prochaine étape de l'expansion germanique : les Sudètes. «La France a dû tolérer l'Anschluss. Pourra-t-elle tolérer, sans déchoir à jamais de son rôle en Europe, sans disparaître de la carte diplomatique du continent, qu'un coup de force soit entrepris contre les frontières ou les institutions de son alliée, la Tchécoslovaquie ? " ${ }^{45}$ Mais, le réquisitoire le plus inattendu vient du Soir : "Il est inévitable, écrit Roland de Marès, que lorsqu'une grande nation marque un tel défaut de confiance en son propre effort, elle voit s'éloigner d'elle les peuples qui, jusque-là, mettaient leur meilleur espoir dans sa volonté de faire respecter les traités et de maintenir la paix. ${ }^{46}$ À ce moment, le Quai d'Orsay a perdu le soutien de son défenseur le plus acharné.

\section{LES DÉSILLUSIONS DE LA GAUCHE}

Comment réagit la gauche socialiste et communiste ? Perçoit-elle un déclin du prestige français sous le Front populaire ? En réalité, son appréciation sera largement conditionnée par la non-intervention dans le conflit espagnol. Celle-ci suscite une réelle déception dans une large majorité de la gauche, exception faite de la tendance socialiste nationale de Paul-Henri Spaak et Henri De Man, de plus en plus influente au sein du parti ouvrier belge (POB). Le "Patron", Émile Vandervelde, est ainsi amené à quitter le gouvernement en janvier 1937 parce qu'il s'oppose aux thèses de cette nouvelle génération. En mai, il adresse une lettre ouverte très amère à Léon Blum. Face à une non-intervention qui échoue, explique-t-il, on voit les libéraux et les travaillistes anglais pousser leur gouvernement à contrer Franco alors que la France reste immobile. "Jusques à quand ce scandale, cette farce ridicule et tragique ? ${ }^{47}$ Les organes de tendance Front populaire, le bruxellois Combat et le liégeois Vigilance reflètent, eux aussi, un embarras évident, alternant reproches et compré-

42. $P P, 21$ janvier 1938, p. 169.

43. $L B, 6$ mars 1938, p. 1.

44. Sur le déclin de ces alliances "de revers", N. Jordan, The Popular Front and Central Europe :

The Dilemmas of French Impotence 1918-1940, Cambridge, 1992.

45. Dernière Heure, 30 mars 1938, p. 1.

46. Soir, 11 avril 1938, p. 1

47. Lettre reproduite dans Le Peuple, $1^{\mathrm{er}}-2$ mai 1937, p. 1. 
hension. Leur base, majoritairement intellectuelle, est en effet très divisée sur la non-intervention.

Sur l'ensemble de la politique extérieure française, un mélange de regrets et d'amertume se dégage donc de la presse socialiste, d'autant plus que les attentes étaient immenses. Au lendemain des élections législatives, Le Peuple, moniteur du POB, entrevoyait le paradis terrestre. Pour Joseph Saxe, dit Jexas, son chroniqueur de politique étrangère, la France se montrerait désormais loyale au Pacte de la SDN en toute occasion ${ }^{48}$. Vigilance semblait tout aussi optimiste ${ }^{49}$. Cependant, quelques points de friction sont déjà perceptibles. L'hommage rendu par Blum aux différents pays voisins ou alliés de la France suscite l'irritation. "Ce passage de la déclaration n'offre guère un grand intérêt. Il est la parfaite démonstration de la solidité des plus déplorables traditions diplomatiques. $»^{50}$ D'autre part, la ratification par le Front populaire de la levée des sanctions prises contre l'Italie en 1935 heurte l'opinion de gauche. Vigilance estime certes qu'on ne doit pas parler de défaite, que Blum, esclave des décisions de Laval, ne pouvait qu'enregistrer une situation acquise ${ }^{51}$, mais Le Peuple se montre beaucoup moins complaisant. Pour Jexas, le gouvernement français «s'est empressé de se rallier à la position anglaise d'abandon $»^{52}$.

Au fil des semaines, la presse socialiste hésite entre critique et justification. Le dramaturge Paul Demasy, correspondant à Paris du quotidien liégeois La Wallonie, raconte le désarroi des petits États d'Europe orientale qui se sont toujours tournés vers l'Occident mais assistent à ses impuissances successives : l'Éthiopie, la Rhénanie, l'Espagne. Dans la droite ligne des gouvernements précédents, celui de Léon Blum choisit la renonciation. Mais, Demasy ne le prend pas pour cible unique : Londres est également coupable ${ }^{53}$.

Le Peuple est, lui aussi, déçu par la France mais il estime que sa diplomatie ne pourrait être mieux dirigée étant donné les circonstances ${ }^{54}$, qu'un autre gouvernement ne pourrait sans doute pas pratiquer une politique différente ${ }^{55}$. C'est la théorie du moindre mal. En mai 1937, le sénateur socialiste Henri Rolin se montrera néanmoins beaucoup plus sévère, au nom de l'idéal genevois dont il est l'éternel défenseur. "Nous manquerions, toutefois, au devoir de franchise le plus élémentaire si nous taisions que la politique extérieure du gouvernement du Front populaire nous a paru souvent mal correspondre à cet idéal. " Rolin dénonce évidemment la non-intervention mais aussi la levée des sanctions contre l'Italie et les efforts déployés afin de ramener Rome, pourtant parjure, à la

48. Ibid., 19 mai 1936, p. 3 .

49. Vigilance, 4 juillet 1936, p. 14.

50. Ibid., p. 15.

51. Ibid.

52. Peuple, 23 juin 1936, p. 1

53. Wallonie, 3-4 octobre 1936, p. 8.

54. Peuple, 22 décembre 1936, p. 3.

55. Ibid., 15 décembre 1936, p. 1. 
SDN. "Ce sont là des inconséquences et des défaillances que le socialisme international en général, et le socialisme belge en particulier, ont douloureusement ressenties ${ }^{56}$ ", s'exclame-t-il. On le voit, socialistes nationaux et internationalistes alternent dans les colonnes du Peuple.

La fin de l'année 1937 et les premiers mois de 1938 se passent dans l'angoisse. La presse socialiste multiplie, en direction de Londres et de Paris, des appels pressants à réagir. Elle insiste sur la crise de confiance à l'Est, sur la menace hitlérienne qui se précise. Le silence des démocraties est "angoissant, tragique, épouvantable ${ }^{57}$, écrit Paul Demasy. "Se taire et capituler quand on représente le droit, le progrès, la raison - tranchons le mot: quand on est la civilisation - et cela parce que l'on n'est pas le plus fort, c'est plus humiliant qu'une défaite $"^{58}$.

Quelques semaines plus tard, c'est à la France seule que s'adresse le rédacteur de La Wallonie, à l'occasion d'une tournée d'Yvon Delbos en Europe centrale et orientale.

"Je comprends seulement que tout ce qui, en Europe, aime encore la liberté, aspire encore à la liberté, a les yeux tournés vers la France et attend d'elle quelque chose $[. .$.$] : l'assurance que la France se lèvera comme un seul homme pour pro-$ téger ou défendre la liberté roumaine, la liberté tchèque, la liberté polonaise, la liberté yougoslave. [...] Je refuse de croire à la fable d'une France bourgeoise et trop prudente, recroquevillée sur elle-même et son bas de laine et qui aurait abdiqué sa mission dans le monde. $"^{59}$

L'Anschluss anéantit tous les espoirs des socialistes. Le Peuple ne fait pas mystère de sa rage : "Vingt ans après la victoire sur l'Allemagne, la France se résigne dans une déchéance humiliante et bientôt tragique. " ${ }^{60}$ Dans La Wallonie, Paul Demasy s'enflamme. "Si la France avait eu un gouvernement assez fort, assez courageux pour dire à temps le mot qu'il fallait, que les Anglais attendaient, pas un soldat allemand n'eût sans doute franchi la frontière autrichienne. Mais, la France n'avait pas de gouvernement, [...] par la faute des "nationaux", des vieux "patriotes". " ${ }^{61}$ Seule la droite, qui vient de refuser le principe de l'union nationale, est considérée comme responsable.

Ce langage ferme tranche avec celui que Demasy avait tenu durant le mois de février. Il plaidait alors l'idée que l'absence de Paris sur la scène internationale était compréhensible et presque excusable. Il expliquait que, grâce au Front populaire, la France était en pleine révolution sociale, en plein bouleversement de son économie, qu'elle menait un « combat non sanglant pour la justice $»^{62}$. Les Français, poursuivait-il, doivent construire une Europe nouvelle chez eux avant de penser à l'Europe. «Ce

56. Peuple, 20 mai 1937, p. 1.

57. Wallonie, $1^{\text {er }}$ septembre 1937 , p. 1.

58. Ibid., p. 6.

59. Ibid., 22 décembre 1937, p. 1.

60. Peuple, 12 avril 1938, p. 3.

61. Wallonie, 23 mars 1938, p. 6

62. Ibid., 16 février 1938, p. 3. 
n'est donc pas le moment de se mettre une guerre sur les bras pour empêcher l'Anschluss. Il y a en France un Anschluss intérieur à réaliser et qui ne pourra réussir sans de profondes réformes de structure. " ${ }^{63}$ Le socialisme national trouvait ici un écho certain.

Si les socialistes ont, dans les premiers mois, tempéré leurs critiques sur le plan de la politique internationale, les communistes ont très rapidement dénoncé, dans leur globalité et sans nuance, les orientations prises par Blum et Delbos. Cette opposition est largement conditionnée par la question espagnole. Dès novembre 1936, La Voix du Peuple, quotidien officiel du PCB, accuse Paris de mettre en péril la démocratie et de servir la réaction.

"Lorsque les communistes français disent que cette politique n'est pas conforme à l'esprit du programme du Rassemblement populaire, ils sont extrêmement modérés dans leurs expressions. Nous n'avons pas les mêmes raisons qu'eux d'employer à l'égard de Léon Blum et de son gouvernement un langage aussi châtié. Et pour dire crûment les choses, nous ne voyons pas en quoi la politique extérieure du gouvernement français differe fondamentalement de celle de notre propre gouvernement que nous ne pouvons qualifier autrement que de trahison nationale. ${ }^{64}$

La rupture est donc très tôt consommée.

Delbos connaîtra néanmoins un court état de grâce en mai 1937. C'est l'époque où il rencontre, à plusieurs reprises mais sans résultat tangible, son homologue russe Litvinov qui souhaite renforcer le Pacte franco-soviétique par un accord militaire ${ }^{65}$. Le reproche fondamental que les communistes adressent à Delbos est de ne pas s'être inscrit dans la ligne diplomatique de Briand. La nomination de Joseph Paul-Boncour dans le second cabinet Blum de mars 1938 est l'occasion d'une dernière flèche empoisonnée : "Paul-Boncour, espérons-le, [...] saura rompre enfin avec la politique funeste de Laval que continuait $\mathrm{M}$. Delbos à l'encontre du programme du Rassemblement populaire. " ${ }^{66}$

La gauche se montre donc relativement sévère à l'égard de la politique extérieure du Front populaire, considérée au mieux comme un moindre mal, une fatalité. Si elle lui semble correspondre à un affaiblissement, il s'agit d'un processus dont les origines remontent à la Grande Guerre mais qui trouve son paroxysme en mars 1938. Le principal reproche adressé au bloc des gauches est de ne pas avoir renversé la vapeur, de ne pas avoir pris d'initiative. Les socialistes nationaux arguent, quant à eux, que la France n'est pas seule en Europe et que les circonstances lui imposaient des concessions.

63. Ibid., 23 février 1938, p. 6.

64. Voix du Peuple, 29 novembre 1936, p. 3.

65. Ibid., 20 mai 1937, p. 3.

66. Ibid., 14 mars 1938, p. 1. 
Évoquer le prestige extérieur d'un État conduit également à poser la question de l'indépendance de sa politique étrangère. Sur ce point, le constat est étonnamment clair et unanime : la Belgique francophone a le sentiment d'une domination britannique sur la France. Londres parait mener le camp des démocraties et exercer une forme de tutelle sur le Quai d'Orsay. Dès 1936, les rapports franco-anglais, qui s'étaient quelque peu distendus en raison du conflit italo-éthiopien et de la remilitarisation de la Rhénanie, se resserrent rapidement. Léon Blum y accorde une importance primordiale et Londres soutiendra avec force son gouvernement. Celui-ci agit de plus en plus et exclusivement en symbiose avec Londres, comme le prouve la non-intervention ${ }^{67}$. D'après François Bédarida, l'influence britannique ne sera jamais aussi forte que sous le cabinet Daladier, à partir d'avril 1938. La France lui semble alors soumise aux exigences d'une "gouvernante anglaise ${ }^{68}$.

Les billets de Roland de Marès dans Le Soir soulignent les rapports de plus en plus cordiaux et intimes entre Paris et Londres ${ }^{69}$ mais n'évoquent, logiquement, aucun lien de sujétion. Il n'est pas question d'accréditer l'idée que le Quai d'Orsay puisse être dirigé ou utilisé par son homologue anglais. Cependant, le ton des articles ne laisse guère de doute sur l'identité du meneur dans le couple franco-britannique. Seule l'Angleterre est en mesure de sermonner et de distribuer les récompenses, comme le prouve notamment un dessin paru à la une du Soir en janvier 1937. Il illustre une phrase d'Anthony Eden, le chef du Foreign Office, manifestement adressée à la France : «Les démocraties ne peuvent devenir le berceau du communisme. $»^{70}$ Le dessin représente une poule, coiffée d'un bonnet phrygien, en train de couver des œufs dont certains sont marqués de la faucille et du marteau. Elle se fait rappeler à l'ordre par un coq superbe, symbolisant la démocratie anglaise... L'anecdote est futile mais significative.

La thématique d'une France à la remorque prend de l'ampleur au fil des semaines et des mois. Pierre Gaxotte ne manque pas de l'exploiter dans ses articles pour La Nation Belge. Disséquant les rapports entre Londres et Paris en octobre 1937, il souligne que la France est considérée comme une simple vassale et ajoute: "On ne parlait pas sur ce ton à M. Poincaré ni même à M. Laval. ${ }^{71}$ Parmi les journaux conservateurs, $L a$ Gazette est sans doute celui qui aura joué le plus fréquemment sur l'image

67. M. Thomas, Britain, France and appeasement: Anglo-French relations in the Popular Front era, New York, 1996.

68. F. Bédarida, La "gouvernante anglaise ", R. Rémond et J. Bourdin (dir.), Édonard Daladier chef de gouvernement (avril 1938 - septembre 1939), Paris, 1977, p. 228-240.

69. Voir, par exemple, Soir, $1^{\text {er }}$ septembre 1936, p. 1, ou 24 avril 1937, p. 1.

70. Ibid., 25 janvier 1937, p. 1.

71. NB, 18 octobre 1937, p. 1. 
d'une domination britannique. En décembre 1936, le quotidien reprend une phrase de Talleyrand: "La France et l'Angleterre doivent être unies comme le cheval et le cavalier. L'essentiel pour la France, c'est de ne pas toujours faire le cheval. ${ }^{72}$ Quelques mois plus tard, la question est évoquée sur un mode moins badin. La Gazette fait d'Yvon Delbos le principal responsable de l'anglophilie démesurée du Front populaire. Elle considère, à tort d'ailleurs, que Léon Blum est davantage soucieux de l'alliance soviétique. Delbos refuse d'accréditer un ambassadeur auprès du roi d'Italie, empereur d'Éthiopie, en arguant que la Grande-Bretagne n'a pas non plus reconnu ce titre. Pour La Gazette, l'explication est « piteuse : n'est-ce pas l'aveu que la politique du Quai d'Orsay est à la remorque du Foreign Office? " ${ }^{73}$. Les expressions pour décrire cette nouvelle constante politique rivalisent d'originalité : "Comme ces snobs qui se font blanchir à Londres, le Quai d'Orsay a pris l'habitude d'aller chercher des directives de l'autre côté du Channel. $»^{74}$

Les communistes et les socialistes approuvent dans l'absolu le rapprochement franco-britannique ${ }^{75}$ mais n'en considèrent pas moins que la balance penche en faveur de Londres. C'est, évidemment, sur la question espagnole que la gauche voudrait voir la France du Front populaire prendre l'initiative. Elle a le sentiment que la Grande-Bretagne met son veto à toute intervention en faveur des Républicains, que Léon Blum seul aurait réagi différemment. En janvier 1937, la Chambre française vote l'interdiction d'envoyer des volontaires en Espagne mais subordonne cette interdiction à une décision similaire des autres puissances. Jexas se réjouit de cette prise de position autonome. "Trop longtemps, on a permis que se créât l'impression qu'en politique extérieure, la France était toujours à la remorque de l'Angleterre. On a trop longtemps attendu une initiative française. ${ }^{76} \mathrm{La}$ satisfaction des gauches est toutefois de courte durée. Dans le conflit espagnol qui s'enlise, La Voix du Peuple fait de l'Angleterre la responsable de l'arrogance allemande et italienne. Elle décrit la France mais également la Belgique comme de vulgaires pantins. «M. Spaak et M. Delbos n'existent plus, ils n'ont plus de voix et seul le ministre des Affaires étrangères de Grande-Bretagne décide et agit. " ${ }^{77}$

Entre les critiques de la droite et celles de la gauche, les libéraux radicaux de La Dernière Heure observent avec une satisfaction non dissimulée que l'harmonisation des diplomaties française et anglaise se fait sous l'égide de Londres dont ils ont presque toujours défendu les orientations. Il n'est pas question d'employer les expressions péjoratives que l'on retrouve dans les journaux conservateurs, socialistes ou commu-

\footnotetext{
72. Gaz, 28 décembre 1936, p. 1.

73. Ibid., 25 février 1937 , p. 1.

74. Ibid., 25 février 1938, p. 1.

75. Voir, entre autres, Peuple, 22 décembre 1936, p. 3

76. Peuple, 19 janvier 1937, p. 1-3.

77. VP, 4 juin 1937, p. 3.
} 
nistes. La France n'est pas «à la remorque " de l'Angleterre, elle s'y est " ralliée ", autant sur la question des négociations avec l'Allemagne ${ }^{78}$ que sur celle de la non-intervention en Espagne ${ }^{79}$. "L'axe de la politique de paix passe aujourd'hui par Londres $"^{80}$, écrit Henry van Leynseele en mai 1937. L'anglophilie des libéraux les conduit donc à minimiser largement le rôle de la France sur la scène européenne, voire à nier sa faculté d'initiative.

La presse n'est pas la seule à penser que le Quai d'Orsay s'aligne sur le Foreign Office. Les courriers diplomatiques du comte de Kerchove, l'ambassadeur belge à Paris, reflètent également cette impression. En novembre 1936, il écrit que la politique de la France est « de plus en plus inféodée à l'Angleterre " ${ }^{81}$. Il emploie même les mots d' "obéissance " et de "passivité " ${ }^{82}$, laissant paraître d'ailleurs une certaine irritation face à cet effacement. Deux mois plus tard, dans un rapport étonnamment optimiste sur la France du Front populaire, il explique que ses soucis monétaires ne sont pas inquiétants, dans la mesure où Léon Blum trouvera toujours en Grande-Bretagne et aux États-Unis les appuis financiers qui lui permettront de les résorber. Pourquoi une telle sollicitude ? "Les milieux anglo-saxons n'ignorent pas qu'à aucune époque de l'histoire de France, un gouvernement n'a été aussi docile, ni aussi soumis à la politique de Londres au point de vue européen, et anglo-saxonne au point de vue mondial. ${ }^{83}$ Le diplomate se garde toutefois de dire que Léon Blum vend la souveraineté française contre un plat de lentilles et insiste plutôt sur une réelle convergence de vues entre le cabinet français et les Anglo-Saxons.

\section{CONCLUSION}

Les Belges francophones les plus idéologiquement marqués à droite ou à gauche jugent donc avec une certaine sévérité la politique de la France en 1936-1938 mais pour des raisons divergentes. Ils s'accordent à déplorer un déclin, une perte de puissance mais n'attribuent pas au Front populaire les mêmes responsabilités. Si les conservateurs insistent sur son influence néfaste, sur son rôle d'accélérateur, la gauche, quant à elle, lui reproche de trahir ses ambitions primitives, d'accepter comme une fatalité le poids des événements. La non-intervention dans le conflit espagnol sous-tend toute son argumentation. Au centre, une large mouvance de l'opinion, rejointe par les socialistes nationaux, prend au contraire appui sur la non-

78. La Dernière Heure, 30 juillet 1936, p. 1.

79. Ibid., 17 janvier 1937 , p. 1.

80. Ibid., 16 mai 1937 , p. 1

81. Archives du ministère des Affaires étrangères belges (ci-après $A M A E B$ ), Correspondance diplomatique. Dossiers "France ", Dossier 538 - 1936, 23 novembre 1936, p. 1.

82. Ibid., p. 5.

83. AMAEB, Dossier 538 - 1937, 29 janvier 1937, p. 6. 
intervention pour juger avec objectivité l'ensemble de l'action internationale du gouvernement français. Mars 1938 sonne cependant le glas des opinions nuancées. La carence française à l'heure où Hitler annexe l'Autriche est trop patente, la menace de guerre trop présente pour que l'angoisse ne l'emporte pas sur la sérénité.

Catherine LANNEAU, Doctorante en histoire, Université de Liège, Belgique. 